\title{
Importance of Graphic Creation in Modern Poster Design
}

\author{
Min Xu \\ Department of Applied Arts, Shanghai Xingjian College, Shanghai, 200027, China
}

Keywords: Poster design; graphic creativity; role

Abstract: Poster design is the most widely used form of visual communication design. Graphics, as the basic element of poster design, plays a central role. The creative process of graphics is a process of creative thinking using visual images. This paper expounds the development, expression form, performance principle and function of graphic creativity in poster design, and expounds the important position of graphic creativity in today's design field.

\section{Introduction}

Poster design, also known as poster design, is the most widely used form of visual communication design. Placard design serves as a medium for visual information transmission, and graphics play a central role as the basic elements of poster design. "A picture is worth a thousand words", just as the contemporary international famous visual designer Horgo? Professor Matisse said: "A good poster should be written in a graphical language rather than a written comment." This shows the important role of graphic creativity in design, how to select and create graphics to express the work The theme is particularly important.

\section{The development of graphic creativity}

The origin of the graphic appears with human beings. The generation of graphics can be traced back to the primitive graphic symbols such as totem patterns and cave paintings in ancient times. These graphics become the medium for communication, communication, expression of emotion and consciousness. This point always runs through every period and stage of the graph from its creation to the present. The form of pictures has been continuously developed and differentiated, gradually developed from the figurative form to the abstract form, and finally evolved into text. This is the first revolution in graphic development. The appearance of words has standardized the symbols and has become an important means of recording and disseminating information. The second revolution was the invention of papermaking and printing in China[1]. Words and drawings are copied in large quantities by means of these two major inventions, making the scope of the dissemination more widespread and the audience more. In particular, the widespread use of printing techniques has promoted the speed of dissemination and aggravated the development of graphics. The third revolution was the industrial revolution in the 19th century. It was an important milestone in the history of graphic development. During this period, many art schools had a profound influence on modern graphics. The invention of the photographic technology and the method of plate making and the innovation of printing technology enabled the dissemination of information. The 
extensiveness has been further expanded. In particular, the invention of photography added new media to graphics and enriched the graphical form. The fourth revolution was an innovative movement of visual art since the 1920s. Among the many schools of art, surrealism had the most profound and direct impact on graphic design. In the surrealist works, the bold deformation of objects and objects The misplaced, inconsistent processing of the material gives surprising visual effects. With the rapid development of science and technology, graphics have become more expressive. Photography, computer and multimedia technology have made the space for graphics more extensive. Designers absorb nutrients in other fields, through graphic refinement in the transmission of information at the same time pay attention to personal style, the speed of graphics beyond the geographical and spatial distance, the faster the spread, a wider range, more information audience.

\section{Graphical Creativity Expressions}

Image. An image is a visual language that visually conveys information, ideas, and exchanges of ideas. It can reproduce things truly, vividly, and objectively. It is particularly advantageous in the instant reproduction of certain objects. With the rapid development of modern science and technology, computer-aided design has entered the design field extensively and has become a widely used tool for designers. Computer graphic synthesis and creative production has become an important expression in modern graphic design[2]. The computer aided design formulas for images are as follows.

$$
\text { Tatget } Q=r+\gamma Q^{\prime}\left(s^{\prime}, \pi\left(s^{\prime} \mid \theta^{\mu^{\prime}}\right) \mid \theta^{Q^{\prime}}\right)
$$

Hand Painted. Decorations, traditional Chinese paintings, illustrations, cartoons, cartoons, etc. all belong to the expression of painting. The painting technique with subjective consciousness fully exerted the author's creativity and artistic talents. Through the original painting language, the author's creative intention was expressed vividly and vividly. The expression technique of painting has a great creative in the design of posters. Space, in creative practice, has a great deal of freedom to express. Due to his painting nature, his works are more appreciated, artistic and original.

Computer graphics. In the design of posters, the computer highlights the characteristics of the software. We use graphic design software to draw the ideal graphics freely. We can represent two-dimensional space and three-dimensional space. Designers can use their imagination to create imagination and creativity. Different graphic languages[3].

\section{Principles of Graphic Creative Expression}

Intuitive. Intuition becomes the dominant trend of graphics in the fierce competition of information dissemination. The graphic ideas tend to be concise, focused, generalized and standardized. They must not only highlight the subject of creation, but also make the picture rich and full.

Symbolic. Through the surface of the graph to dig deeper meanings, feel the inner meaning. The symbolic graphic language can enrich the graphic content and make the graphic more cultural.

Inspirational. Poster design has the strongest purpose. Simple graphic ideas will convey deep social philosophies and philosophy of life. It will play an important role in aesthetic education. Accurate and clear graphic language will impress the hearts of the audience and cause strong emotional resonance. Cause people to think deeply[4].

Personalize. In the context of various information interactions, it is necessary to strengthen the personal expression of graphic design in order to instantly attract the viewer's attention. The graphic 
with personalized features can give people a fresh visual experience, enhance the visual impact, and play an unexpected effect. At the same time, it also meets the psychology of modern people's innovation and variation, and it is more suitable for the performance of visual communication.

\section{The role of graphic creativity in the design of posters}

\subsection{Information transfer}

Graphics, text, and color are three elements of poster design. In the design of posters, graphics are the most important of the three elements in a work. Whether a graphic idea can express the theme correctly will directly affect the transmission and communication of information. Graphic creativity is a visual center that attracts people's attention in poster design work. An excellent placard design work often expresses the theme of the creation accurately and clearly in a unique graphic language. The theme with rich connotation is represented by the most concise visual elements. After viewing the graphic with the eyes, the author's intention can be understood. This is the rich charm of graphics. Good graphics can indeed be called "worldwide language." The ideology in the art of poster design can be said to be the most penetrating form, and many poster designs include people's perspectives, values, and spiritual orientations. As the medium closest to people's lives, posters convey many different ideologies such as living beliefs, spiritual ideas, value judgments, and behavioral standards. Graphic arts usually make people feel psychological through strong images and stunning visual effects. Consensus has emerged to reveal the connotation of the topic, triggering emotional resonance, attracting people's attention, and inspiring people's sense of reading. It is more memorable than words, and more subtle and profound than words. In order to achieve rapid, accurate and effective dissemination of information. The expression of creative graphic semantics in the design of posters not only conveys the rational appeal of the designers themselves, but also allows the audience to produce a rich association space, feel the pleasure brought by the design, the aesthetic and the guidance of the information, in the sense of resonance and emotion The demand for automation is generated. At this time, the semantics of creative graphics in poster design are implicitly recognized and disseminated by the audience. Graphic creative information transmission effect as shown below.

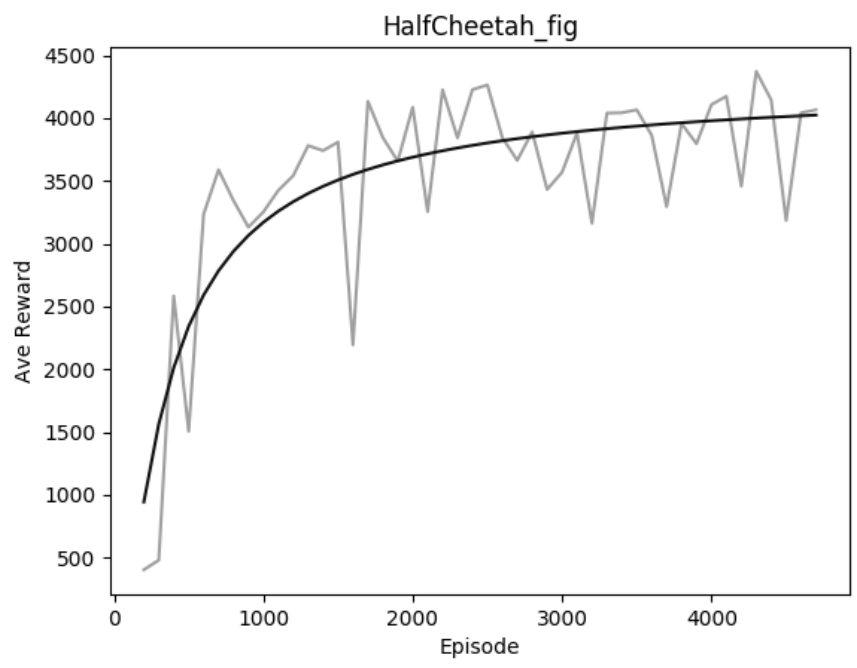

Fig.1 Graphic creative information transmission effect 


\subsection{Visual impact}

Semantic communication of graphic design in poster design has become the focus of designers' attention. It has added a lot of means in the form of expression to make it more colorful and effectively convey the intention of the designer. In the process of realizing graphic creativity, one must be good at discovering what others have not discovered, showing what others have not demonstrated, and associating other people to think of others, and imagining a variety of combinations that people have never imagined. Only in this way can graphic design eventually achieve effective dissemination of information, which has extensive implications for social development, information exchange, and concept communication. To make graphic design stand out in numerous information exchanges, the important principle is the unique creativity and perfect and accurate graphical representation. This requires that we must be unique in the design and creative ideas in the design practice. The vision, the new visual experience, is attractive in a unique form of expression and emphasizes the visual appeal. From the simple graphic symbols, we can realize the deep meaning and cultural connotation. This is the basic task given by the visual communication. With the development of the times and the constant updating of design methods, modern graphic design is no longer the sensibility of simple visual factors. The choice is not simply a decorative composition in design, but is based on the deeper levels of research, understanding, and creation of human visual experience, psychological experience, and behavioral activity. Therefore, the influential graphic is often derived from life and a perceptual understanding of life. In the creative process, with the help of the original sensuous object, we create a new, original image that humans have never felt before.

\section{Summary}

In summary, graphic creativity is a process that has been refined, processed, and created. Graphics are the focus of the thematic expression of poster design works, and they are also the most intuitive and highly focused visual focus. Novel, innovative, and accurate graphics will impress people's hearts, and they can produce emotional resonance in the mind. They can visually transmit information to the outside world and achieve the role of communication. Creative ideas combined with creative themes from the graphics of the form of expression, performance principles in-depth exploration of creative ideas, sublimation of creative elements. Finally, a new graphic language that meets the needs of modern aesthetics is formed. Therefore, designers must continue to innovate, so that graphics in the design of posters continue to play a central role with the development of the times.

\section{References}

[1] Iztok Fister,Matjaž Perc,Karin Ljubič,Salahuddin M. Kamal,Andres Iglesias,Iztok Fister. Particle swarm optimization for automatic creation of complex graphic characters[J]. Chaos, Solitons and Fractals: the interdisciplinary journal of Nonlinear Science, and Nonequilibrium and Complex Phenomena,2015,73.

[2] Pedro Beirão,Duarte Valério. Creation of a Virtual Graphic Interface Applied to a Process Control System[J]. Procedia - Social and Behavioral Sciences,2012,46.

[3] Anonymous. Beyond.com; GRAPHIC U.S. Job Creation Stagnant in August, Yet Some Industries Maintain Strong Hiring Activity [J]. Computers, Networks \&amp; Communications,2011.

[4] I. A. Dychka,M. V. Novosad,T. Yu. Grybok. Data conversion in creation and processing of multicolored graphic codes [J]. Radioelectronics and Communications Systems,2013,56(7). 